\title{
HIGGS SEARCHES AT ATLAS
}

Carlos Solans on behalf of the ATLAS Collaboration ${ }^{a}$ Instituto de Física Corpuscular (IFIC), Centro Mixto Universidad de Valencia CSIC, Valencia, Spain

Abstract.We review the current searches at ATLAS for Higgs bosons in the Standard Model performed at the LHC with a center of mass energy of $7 \mathrm{TeV}$. Results from pp-collision data with an integrated luminosity of up to $2.3 \mathrm{fb}^{-1}$ are discussed.

\section{Introduction}

The discovery of the mechanism for electroweak symmetry breaking is one of the goals of the physics programme at the Large Hadron Collider (LHC). In the Standard Model (SM), this is achieved by invoking what is known as the Higgs mechanism, leading to the production of the Higgs boson [1,2]. The Higgs boson mass, $m_{H}$, is an unknown parameter in the SM, although it is weakly coupled to other observables. The ATLAS [3] collaboration has carried out Higgs boson searches in different channels with $p p$ collision data at a centreof-mass energy of $7 \mathrm{TeV}$ with an integrated luminosity of up to $2.3 \mathrm{fb}^{-1}$. The systematic uncertainty of the luminosity measurement is estimated to be $3.4 \%$. Exclusion limit results are presented using CLs technique [4,5].

\section{SM Higgs boson search channels in ATLAS}

The different SM Higgs boson searches carried out in ATLAS are briefly described in the following paragraphs. Some of them already provide exclusion results for the integrated luminosity analysed, as shown in Figure 1.

The $H \rightarrow \gamma \gamma[6]$ analysis is carried out for Higgs boson mass hypotheses between 110 and $150 \mathrm{GeV} / c^{2}$. Selected events are separated into five independent channels based on the direction in which each photon was emitted and its photon to electron-pair conversion status. The background is estimated from an unbinned fit of the diphoton invariant mass spectrum. Total integrated luminosity analysed in this channel is $1.08 \mathrm{fb}^{-1}$.

The $H \rightarrow \tau \tau \rightarrow \ell \tau_{\text {had }}+3 \nu[7]$ (semi-leptonic) and $H \rightarrow \tau \tau \rightarrow \ell^{+} \ell^{-}+4 \nu[8]$ (fully leptonic) searches are performed as a counting analysis for Higgs boson mass hypotheses between 110 and $150 \mathrm{GeV} / c^{2}$. These events are required to have one or two high $p_{T}$ leptons in the final state and a jet with transverse momentum that exceeds $40 \mathrm{GeV}$. The resulting boost of the Higgs boson implies a large missing transverse energy in the event, which, allows for a good discrimination against $Z / \gamma \star \rightarrow \ell \ell, Z \rightarrow \tau \tau$ and QCD multi-jet backgrounds. The top-quark pair production and all other backgrounds are taken from the simulation, except for the QCD background with jets faking leptons which is

\footnotetext{
${ }^{a}$ e-mail: carlos.solans@cern.ch
} 


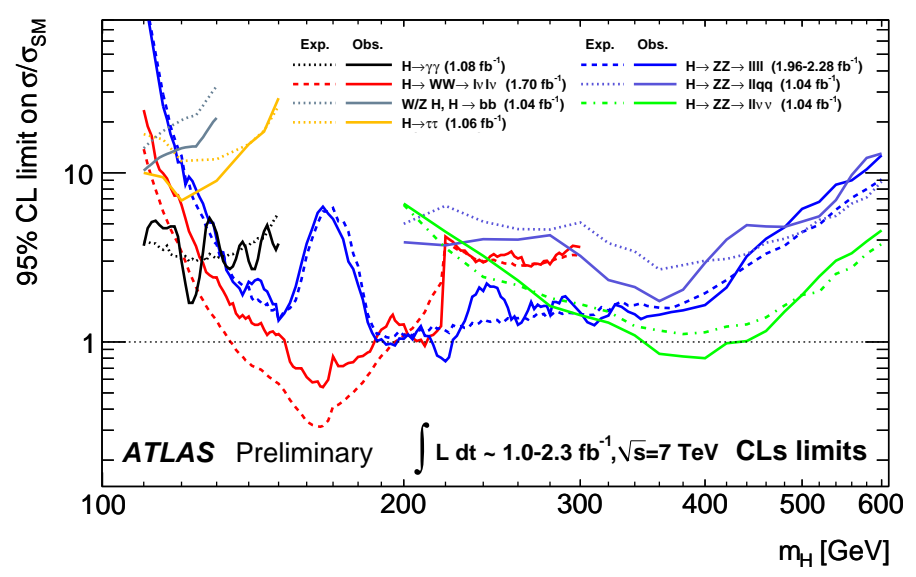

Figure 1: Observed and expected 95\% C.L. upper limits on the signal strength modifier $\mu=$ $\sigma / \sigma_{S M}$, obtained with the CLs method, for the different SM Higgs searches in ATLAS, as a function of the SM Higgs boson mass in the range $110-600 \mathrm{GeV} / c^{2}$.

estimated within an independent control sample. Analysis is carried out with a dataset corresponding to an integrated luminosity of $1.06 \mathrm{fb}^{-1}$.

The $H \rightarrow W W^{\star} \rightarrow \ell \nu \ell \nu$ [9] search is performed as a counting analysis for Higgs boson mass hypotheses between 110 and $300 \mathrm{GeV} / c^{2}$, for an integrated luminosity of $1.7 \mathrm{fb}^{-1}$. The main background contributions are estimated from the data using control regions. The analysis is separated into 0-jet and 1-jet channels. The systematic uncertainty on the $W W$ background estimate comes mainly from the low number of counts, and to the uncertainty on the extrapolation from theoretical scale variations and jet energy scale uncertainties. There are significant uncertainties considered on the top-quark background in both the 0-jet channel and 1-jet channels. The other backgrounds are also measured in control samples of data, except for the small contributions of $W Z$ and $Z Z$ that are taken from simulation. The discriminant is the transverse mass distribution, and the event selection is optimised in three mass regions $\left(<170 \mathrm{GeV} / c^{2}, 170-220 \mathrm{GeV} / c^{2}\right.$, and $\left.>200 \mathrm{GeV} / c^{2} c\right)$ with different signal and background control regions.

The $H \rightarrow Z Z^{\star} \rightarrow \ell \ell \ell \ell[10]$ search is performed for Higgs boson mass hypotheses in the range from 110 to $600 \mathrm{GeV} / c^{2}$, selecting three distinct final states: $4 \mu, 2 e 2 \mu$ and $4 e$. The integrated luminosity for each final state is $2.3 \mathrm{fb}^{-1}$, $2.0 \mathrm{fb}^{-1}$ and $2.0 \mathrm{fb}^{-1}$ respectively. The main background of this search is the $Z Z^{\star}$ production, and is estimated from simulation. The reducible $Z+$ jets background is estimated from control regions in data.The final discriminating variable is the invariant mass distribution of the selected events. 
The $H \rightarrow Z Z \rightarrow \ell \ell \nu \nu[11]$ analysis is carried out for the 200 to $600 \mathrm{GeV} / c^{2}$ range of Higgs boson mass hypotheses, and divided in a high $\left(\geq 280 \mathrm{GeV} / c^{2}\right)$ and a low mass region. A total integrated luminosity of $1.04 \mathrm{fb}^{-1}$ is considered. This search is orthogonal with respect to the $H \rightarrow W W^{\star} \rightarrow \ell \nu \ell \nu$ search by design. Diboson production, $Z Z, W W$ and $W Z$, is the main background to this search for high transverse mass values. Its normalisation uncertainty is taken from theory. Normalisation uncertainties for $\mathrm{Z}+$ jets, $\mathrm{W}+$ jets, top and QCD-multijet production are estimated from data. The final discriminating variable is the transverse mass of the dilepton plus missing momentum system, where the missing momentum is assumed to originate from a Z-boson decay.

The $H \rightarrow Z Z \rightarrow \ell \ell q q[12]$ analysis is performed for Higgs boson mass hypotheses in the 200 to $600 \mathrm{GeV} / c^{2}$ range, divided into two regions: below and above $300 \mathrm{GeV} / c^{2}$. A total integrated luminosity of $1.04 \mathrm{fb}^{-1}$ is used. The final state is separated into two categories: the tagged analysis where the two jets are b-tagged and the untagged analysis where at most one of the jets is btagged. The dominant background in this analysis is $\mathrm{Z}+$ jets production, which is normalised from a control region defined by the sidebands of the dijet mass distribution. The other backgrounds are estimated with the simulation verified with data control samples for the top-quark background. The dilepton-dijet invariant mass is used as the final discriminant and its shape is estimated using simulation for both the signal and the backgrounds.

The $H \rightarrow \bar{b} b[13]$ analysis combines $W H(\ell \nu \bar{b} b)$ and $Z H\left(\ell^{+} \ell^{-} \bar{b} b\right)$ searches for the Higgs boson in the mass range between 110 and $130 \mathrm{GeV} / c^{2}$ for an integrated luminosity of $1.04 \mathrm{fb}^{-1}$. In the $\ell \nu \bar{b} b$ channel, the dominant backgrounds arise from top-quark production and $W+$ jets. The contribution from the multi-jet, top and $\mathrm{W}+$ jets backgrounds is determined by a simultaneous template fit to control regions of data. In the $\ell^{+} \ell^{-} \bar{b} b$ channel, the dominant background comes from $\mathrm{Z}+$ jets production and is estimated using a control region of data for normalisation and MC simulations for a shape template. Other sources of background are estimated by MC simulation. The discriminant is the invariant mass distribution of the $\bar{b} b$ pairs.

\section{3 Combined SM Higgs boson search in ATLAS} experiment is presented in Figure 2, where the CLs value for the SM Higgs boson hypothesis is shown as a function of the Higgs boson mass in the range

$94 \quad 110-600 \mathrm{GeV} / c^{2}$. The SM Higgs boson is excluded at $95 \%$ C.L. in the mass 95 range $145-231 \mathrm{GeV} / c^{2}, 254-281 \mathrm{GeV} / c^{2}$, and $294-466 \mathrm{GeV} / c^{2}$. 


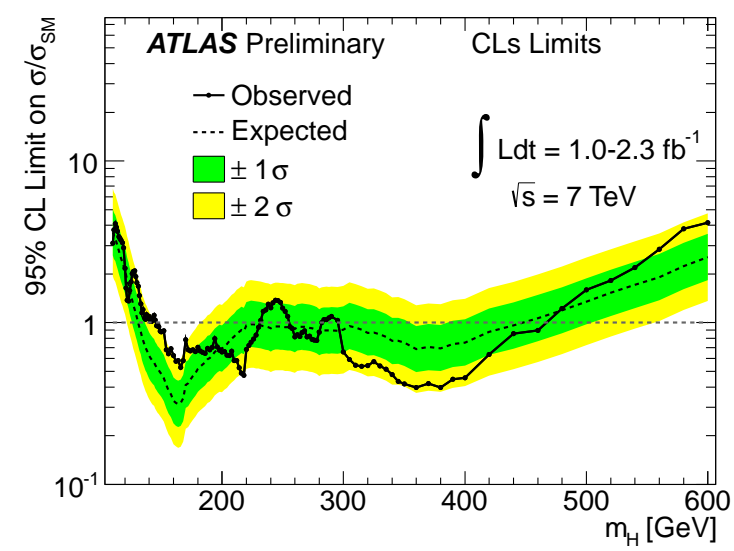

Figure 2: ATLAS combined 95\% C.L. upper limits on the signal strength modifier $\mu=$ $\sigma / \sigma_{S M}$, obtained with the CLs method, as a function of the SM Higgs boson mass in the range $110-600 \mathrm{GeV} / c^{2}$.

\section{Conclusions}

SM Higgs searches in ATLAS are described. Individual and combined results with up to $2.3 \mathrm{fb}^{-1}$ of integrated luminosity are presented. A large exclusion band is placed over the production of a SM Higgs boson with the amount of data analysed.

\section{References}

[1] P.W. Higgs. Broken symmetries, massless particles and gauge fields. Phys. Lett., 12:132133, 1964.

[2] P.W. Higgs. Broken symmetries and the masses of gauge bosons. Phys. Rev. Lett., 13:508509, 196.

[3] ATLAS Collaboration. The ATLAS Experiment at the CERN Large Hadron Collider. JINST, 3:S08003, 200

[4] A. L. Read. Presentation of search results: The CL(s) technique. J. Phys., G28:26932704, 2002.

[5] G. Cowan, K. Cranmer, E. Gross and O. Vitells. Asymptotic formulae for likelihood- based tests of new physics. Eur. Phys. J., C71:119, 2011.

[6] ATLAS Collaboration Search for the Standard Model Higgs boson in the two photon decay channel with the ATLAS detector at the LHC. CERNPH-EP-2011-129, arXiv:1108.5895.

[7] ATLAS Collaboration Search for neutral MSSM Higgs bosons de- 
caying to tau+tau- pairs in proton-proton collisions at sqrt(s) $=7 \mathrm{TeV}$ with the ATLAS detector. ATLAS-CONF-2011-132, http://cdsweb.cern.ch/record/1383835

[8] ATLAS Collaboration Search for the Standard Model Higgs boson in the decay mode $H->$ tau + tau- $->l l+4$ neutrinos in Association with jets in Proton-Proton Collisions at sqrt(s) $=7$ TeV with the ATLAS detector. ATLAS-CONF-2011-133, http://cdsweb.cern.ch/record/1383836

[9] ATLAS Collaboration Search for the Standard Model Higgs boson in the $H \rightarrow W W^{(*)} \rightarrow \ell \nu \ell \nu$ decay mode using $1.7 \mathrm{fb}^{-1}$ of data collected with the ATLAS detector at $\sqrt{s}=7$ TeV ATLAS-CONF-2011-134, http://cdsweb.cern.ch/record/1383837

[10] ATLAS Collaboration Search for the Standard Model Higgs boson in the decay channel $H \rightarrow Z Z^{(*)} \rightarrow 4 l$ with the ATLAS detector. CERN-PHEP-2011-144, arXiv:1109.5945.

[11] ATLAS Collaboration Search for a Standard Model Higgs boson in the $H \rightarrow Z Z \rightarrow l^{+} l^{-} \nu \bar{\nu}$ decay channel with the ATLAS detector. CERNPH-EP-2011-142, arXiv:1109.3357.

[12] ATLAS Collaboration Search for a heavy Standard Model Higgs boson in the channel $H \rightarrow Z Z \rightarrow l^{+} l^{-} q \bar{q}$ using the ATLAS detector. CERN-PHEP-2011-136, arXiv:1108.5064.

[13] ATLAS Collaboration Search for the Standard Model Higgs boson produced in association with a vector boson and decaying to a b-quark pair with the ATLAS detector at the LHC. ATLAS-CONF-2011-103, http://cdsweb.cern.ch/record/1369826

[14] ATLAS Collaboration Update of the Combination of Higgs Boson Searches in $p p$ Collisions at sqrt(s) $=7 \mathrm{TeV}$ with the ATLAS Experiment at the LHC. ATLAS-CONF-2011-135, http://cdsweb.cern.ch/record/1383838 